\title{
Captura e Conversão de Dióxido de Carbono: a Promoção de um Resíduo a Produto de Valor Acrescentado
} Sara Realista

\begin{abstract}
Capture and Conversion of Carbon Dioxide: the Promotion of a Waste to an Added Value Product. Climate change has been one of the most discussed issues of our society. The main character of this novel has been carbon dioxide that became a villain when its atmosphere concentration reached dangerous values, affecting Earth's thermal stability. The scientific community gathered forces to develop more sustainable methods to convert this molecule into others that are useful to fulfil society's needs. During this work, new catalysts were synthesised to convert $\mathrm{CO}_{2}$ using visible light. Catalyst design allowed to obtain methane, one of the main components of natural gas, from the $\mathrm{CO}_{2}$ conversion using visible light.
\end{abstract}

\begin{abstract}
As alterações climáticas são um dos temas mais debatidos nos dias de hoje. O principal ator desta novela tem sido o dióxido de carbono $\left(\mathrm{CO}_{2}\right)$ que, quando começou a atingir níveis perigosos na atmosfera, passou a vilão afetando a estabilidade térmica da Terra. A comunidade científica uniu esforços e começou a pensar em vias sustentáveis para converter esta molécula noutras que podem ser úteis para satisfazer as necessidades da sociedade. Neste trabalho, desenvolveram-se catalisadores para a conversão de $\mathrm{CO}_{2}$ ativada por luz visível. Através do desenho dos catalisadores foi possível detetar a formação de metano, que é um dos componentes maioritários do gás natural, através da conversão de $\mathrm{CO}_{2}$ com luz.
\end{abstract}

\section{$\mathrm{CO}_{2}$ e as alterações climáticas}

Nos últimos tempos, muito se tem falado das emissões de $\mathrm{CO}_{2}$ e das suas consequências para o planeta Terra. Aquecimento global e alterações climáticas têm vindo a ser debatidas pelo mundo junto da sociedade civil e política com o intuito de se tomarem ações que possam mitigar este problema. Antes de se mergulhar nas soluções propostas por vários cientistas em todo o mundo, dever-se-á primeiro refletir sobre o $\mathrm{CO}_{2} \mathrm{e}$ o seu papel nesta temática.

O $\mathrm{CO}_{2}$ é um dos principais intervenientes no ciclo de carbono (Figura 1) que é constituído pelos vários reservatórios de carbono (rochas, oceanos, solo, atmosfera) e pelos fluxos que existem entre eles.
Estes fluxos ocorrem devido a diferentes processos físicos, químicos, geológicos e biológicos, e quando em equilíbrio ajudam a manter a temperatura da Terra estável. $0 \mathrm{CO}_{2}$ participa ativamente neste ciclo, principalmente por ser um gás de efeito de estufa, o que faz com que aprisione parte da energia que provém do sol, mantendo a temperatura terreste ideal para o desenvolvimento de seres vivos. Qualquer desequilíbrio provocado neste ciclo leva a que a temperatura na Terra se altere com consequentes efeitos drásticos e irreversíveis, como por exemplo, o degelo dos glaciares levando ao aumento do nível médio dos oceanos [1]. 


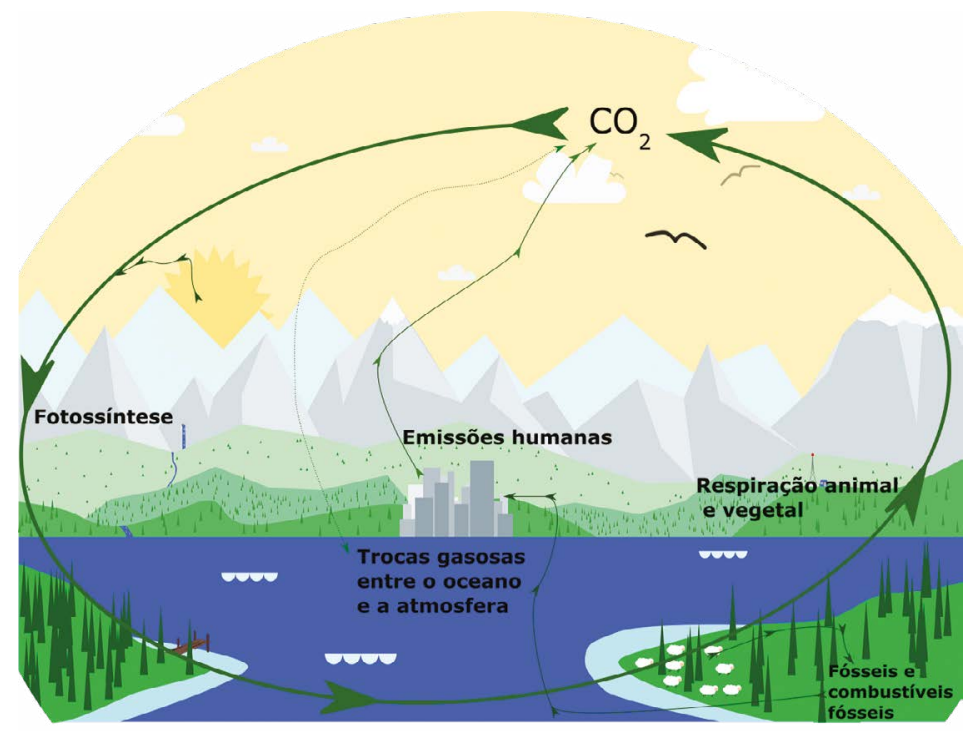

Figura 1 - Os vários reservatórios de carbono e os fluxos entre eles que constituem o ciclo de carbono.

Este desequilíbrio tem sido maioritariamente provocado pelo Homem, e foi depois de 1950, quando decorreu a terceira revolução industrial, que os níveis de $\mathrm{CO}_{2}$ começaram a aumentar até aos níveis perigosos dos dias de hoje (Figura 2) - aumentaram de 280 ppm (partes por milhão) para mais de 420 ppm de $\mathrm{CO}_{2}$ na atmosfera e continuam a subir [2]!

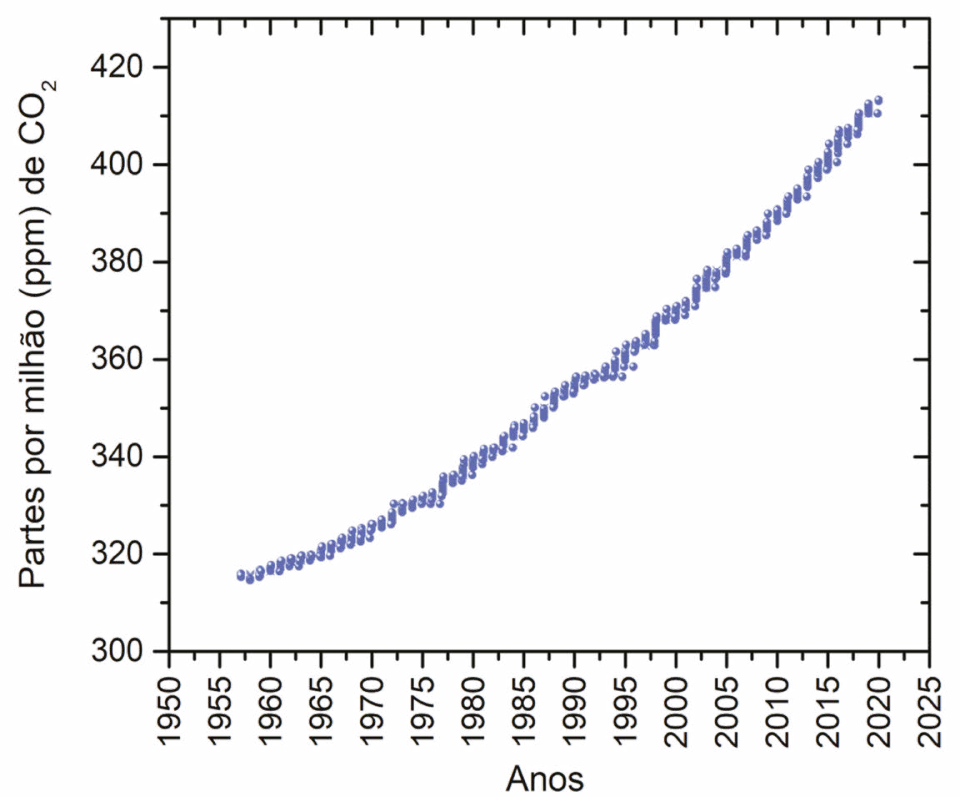

Figura 2 - Níveis atmosféricos de $\mathrm{CO}_{2}$ medidos no

Observatório de Mauna Loa no Havai. 0 gráfico mostra apenas os resultados depois do momento em que os níveis de $\mathrm{CO}_{2}$ iniciaram a sua subida nunca vista. 0 início desta subida é coincidente com o início da terceira revolução industrial. Os dados para construção do gráfico foram retirados do site da NASA (Administração Nacional de Aeronáutica e Espaço, Estados Unidos da América).

\section{Um para todos e todos para um!}

Com o aumento da concentração de $\mathrm{CO}_{2}$ na atmosfera, tornou-se necessário desenvolver novos materiais e métodos para a remoção deste gás da atmosfera, surgindo a possibilidade da sua captura e/ou conversão em produtos que possam ser reutilizados pela sociedade. Já existem alguns exemplos do uso de $\mathrm{CO}_{2}$ na indústria, nomeadamente no processamento de alimentos, como por exemplo, no fabrico de refrigerantes. Mas os cientistas querem ir mais longe! Querem poder utilizar este gás como fonte de carbono para a obtenção de outros químicos que possam ser usados como combustíveis, fármacos, entre outros [3].

Numa primeira instância, em quê e como é que pode ser convertido o $\mathrm{CO}_{2}$ ? $\mathrm{O} \mathrm{CO}_{2}$ pode ser convertido em monóxido de carbono (CO), ácido fórmico $(\mathrm{HCOOH})$, formaldeído $(\mathrm{HCHO})$, metanol $\left(\mathrm{CH}_{3} \mathrm{OH}\right)$ e metano $\left(\mathrm{CH}_{4}\right)$, através da sua redução mediada por protões e eletrões (Equações 1-5).

$$
\begin{aligned}
& \mathrm{CO}_{2}+2 \mathrm{H}^{+}+2 \mathrm{e}^{-} \rightarrow \mathrm{CO}+\mathrm{H}_{2} \mathrm{O} \\
& \mathrm{CO}_{2}+2 \mathrm{H}^{+}+2 \mathrm{e}^{-} \rightarrow \mathrm{HCOOH} \\
& \mathrm{CO}_{2}+4 \mathrm{H}^{+}+4 \mathrm{e}^{-} \rightarrow \mathrm{HCHO}+\mathrm{H}_{2} \mathrm{O} \\
& \mathrm{CO}_{2}+6 \mathrm{H}^{+}+6 \mathrm{e}^{-} \rightarrow \mathrm{CH}_{3} \mathrm{OH}+\mathrm{H}_{2} \mathrm{O} \\
& \mathrm{CO}_{2}+8 \mathrm{H}^{+}+8 \mathrm{e}^{-} \rightarrow \mathrm{CH}_{4}+2 \mathrm{H}_{2} \mathrm{O}
\end{aligned}
$$

Contudo, a obtenção destes diferentes produtos depende do número de eletrões e de protões necessários para obter cada um, sendo mais favorável a produção de $\mathrm{CO}$, que necessita apenas de dois eletrões e dois protões, do que a produção de $\mathrm{CH}_{4}$ para a qual são precisos oito eletrões e oito protões [4]. A conversão de $\mathrm{CO}_{2}$ é um grande desafio não só pelo desejo de obter apenas um produto entre os vários que podem ser obtidos, mas também pela existência de reações competitivas, como a redução do protão a hidrogénio molecular $\left(\mathrm{H}_{2}\right)$. É neste momento que entram moléculas com metais na sua estrutura (compostos de coordenação) que têm a possibilidade de ser desenhadas e sintetizadas especificamente para serem mais seletivas, ou seja, originar apenas um produto pela conversão de $\mathrm{CO}_{2}[5]$.

A catálise tem sido uma das abordagens mais frequentes à conversão de $\mathrm{CO}_{2}$, porque permite o uso de um composto (catalisador) que acelera uma reação sem ser consumido. Os compostos de coordenação têm sido usados como catalisadores ao longo dos anos para inúmeras transformações químicas, como por exemplo, no processo de Monsanto para a formação de ácido acético (usado na produção de 
garrafas de plástico). A fotorredução (Figura 3, via A) e a eletrorredução (Figura 3, via B) catalisada de $\mathrm{CO}_{2}$ têm sido as metodologias mais aplicadas para esta reação e surgem como alternativas mais verdes e fáceis de manipular quando comparadas com a catálise química. Como mencionado anteriormente, a conversão de $\mathrm{CO}_{2}$ é mediada por eletrões que na via A são provenientes de uma molécula que os fornece após exposição à luz, enquanto que na via B é a energia elétrica que liberta os eletrões. Os eletrões ativam os catalisadores (Figura 3) que depois vão converter $\mathrm{O}_{\mathrm{CO}_{2}}$ em moléculas que podem ser utilizadas para satisfazer direta- ou indiretamente as necessidades da sociedade moderna, como por exemplo, em combustíveis, na alimentação, na síntese de fármacos, etc. [6].

\section{$\mathrm{CO}_{2}$ vê a luz - conversão de $\mathrm{CO}_{2}$ através de luz visível}

A fotorredução de $\mathrm{CO}_{2}$ tem sido uma via bastante atrativa tendo em conta que em última instância a luz solar pode ser usada como iniciadora do processo catalítico. Encontrar catalisadores que sejam seletivos para a conversão de $\mathrm{CO}_{2}$ através da luz visível foi o objetivo do trabalho desenvolvido durante o projeto de doutoramento.

A escolha recaiu em catalisadores à base de criptandos. Jean-Marie Lehn foi o investigador responsável pelo nascimento destes compostos e o seu desenvolvimento valeu-Ihe o prémio Nobel da Química em 1987 [7]. Os criptandos são moléculas orgânicas bicíclicas contendo átomos doadores de nitrogénio ou oxigénio (círculos verdes na Figura 4) que se assemelham a uma "gaiola". Estas gaiolas coordenam aos metais originando os criptatos (Figura 4). Depois da formação do criptato, podem acomodar-se uma grande variedade de aniões (iões com carga negativa). Esta "gaiola" pode ser modificada de forma a aprisionar diferentes tipos de aniões, através da modificação da flexibilidade dos braços do criptando e da distância entre os metais.

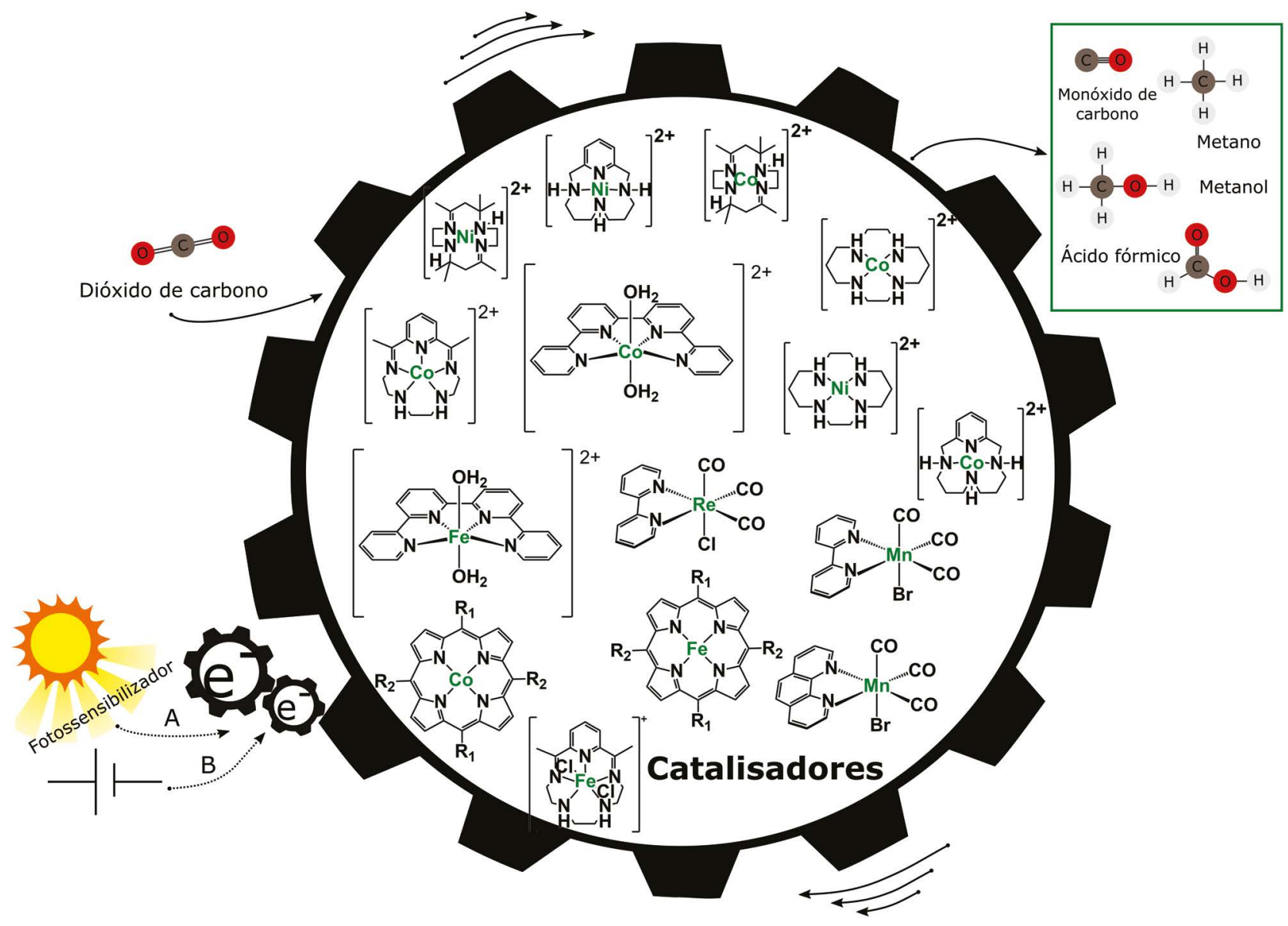


Jane Nelson e seus colaboradores [8] descobriram que os criptandos da Figura 5 são capazes de capturar $\mathrm{CO}_{2} \mathrm{da}$ atmosfera e convertê-lo em anião bicarbonato $\left(\mathrm{HCO}_{3}{ }^{-}\right)$depois da coordenação com diferentes metais: cobalto (Co), zinco (Zn), níquel (Ni) e cobre (Cu). De facto, estes compostos seguem o mecanismo adotado pelas anidrases carbónicas, que se trata de uma família de enzimas responsáveis pela conversão de $\mathrm{CO}_{2}$ no anião bicarbonato em humanos, bactérias, algáas, plantas, entre outros [9]. Esta semelhança demonstra o potencial deste tipo de compostos para mimetizar processos já encontrados na natureza!

Mais tarde Moller e a sua equipa $[10,11]$ desvendaram que alterações nas estruturas destes criptatos (Figura 5) tinham influências dramáticas na seletividade para determinados aniões. Neste projeto pretendeu-se entender esta influência tanto na captura de $\mathrm{CO}_{2}$ como na sua conversão em produtos de valor acrescentado. Assim prepararam-se quatro criptatos de cobalto diferentes que estão representados na Figura 6. Durante o trabalho que estava a ser realizado no âmbito deste projeto [12], Zhong, Lu e seus colaboradores [13] publicaram um criptato de cobalto semelhante, capaz de converter com elevada eficiência e seletividade $\mathrm{CO}_{2}$ a $\mathrm{CO}$ usando luz visível (LED azuis). O CO não pode ser usado diretamente pela sociedade, mas pode ser usado como precursor na síntese de combustíveis ou de fármacos.

Figura 4 - Criptandos e criptatos. As linhas verdes representam os "braços" do criptando que contêm na sua estrutura átomos doadores ( $\mathrm{N}$ e 0 , estes últimos representados por círculos verdes) que vão coordenar metais. Após a coordenação do metal ao criptando, originando um criptato, este pode acomodar diferentes aniões entre os dois centros metálicos com elevada seletividade.

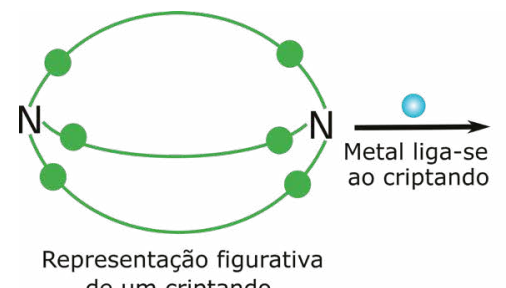
de um criptando

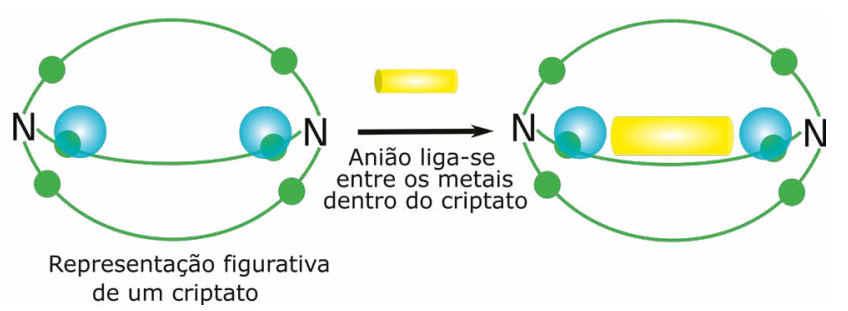

de um criptato

Figura 5 - Os criptandos sintetizados por Jane Nelson e seus colaboradores que são capazes de capturar $\mathrm{CO}_{2}$ da atmosfera e convertê-lo em anião bicarbonato $\left(\mathrm{HCO}_{3}^{-}\right)$[8].

\section{Criptando}<smiles>c1cc2cc(c1)CNCCN1CCNCc3cccc(c3)CNCN(CCNC2)CCNCc2cccc(c2)C1</smiles>

Criptato

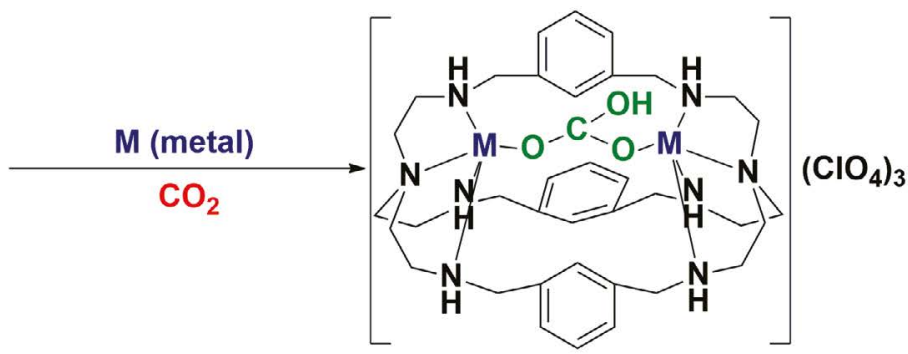

$\mathrm{M}=\mathrm{Co}, \mathrm{Cu}, \mathrm{Zn}, \mathrm{Ni}$

$A$ verde $o$ anião bicarbonato 
Figura 6 - Criptatos de Co estudados neste trabalho de doutoramento. Alterouse o substituinte do anel aromático (R) por diferentes grupos químicos de forma a estudar a sua influência na captura e conversão de $\mathrm{CO}_{2}[12]$.

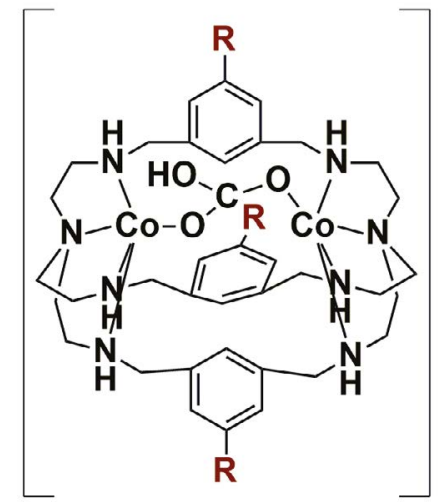

$\left(\mathrm{ClO}_{4}\right)_{3}$

Alterações à estrutura do criptato através da modificação do substituinte R que deu origem a quatro criptatos diferentes: Criptato 1: $\mathrm{R}=$ hidrogénio Criptato 2: $\mathrm{R}=$ bromo Criptato $3: R=$ nitro Criptato 4: $\mathrm{R}=$ acetileno

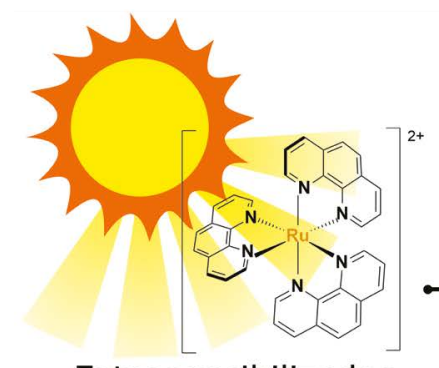

Fotossensibilizador fotoconversão de $\mathrm{CO}_{2}$ catalisada pelos criptatos sintetizados no trabalho de doutoramento [12].

Assim, foram usados os criptatos da Figura 6 nas mesmas condições de fotoconversão de $\mathrm{CO}_{2}$ utilizada pela equipa de Zhong e Lu e avaliado o seu potencial. $\mathrm{Na}$ Figura 7 está representada uma imagem explicativa do processo catalisado por luz visível. A reação foi realizada numa mistura de solvente orgânico e água, utilizando um composto de ruténio como fotossensibilizador (composto que recebe a luz e transfere eletrões para o catalisador) e trietanolamina como dador sacrificial de protões (Figura 7). A conversão de $\mathrm{CO}_{2}$ não seria possível sem a presença do catalisador criptato, que se encontra na reação na concentração de 25 nanomolar, que corresponde a ter uma quantidade 1000 milhões de vezes mais pequena do que 1 miligrama dissolvidas em 1 mililitro! É um valor extremamente baixo comparativamente com outros catalisadores já utilizados nesta reação. Espantosamente, devido à modificação da estrutura dos criptatos conseguiu-se detetar não só CO, mas também $\mathrm{CH}_{4}$ que pode ser utilizado diretamente pela sociedade sendo que é o componente maioritário do gás natural.
0 trabalho realizado neste projeto deu origem a uma publicação que se tornou capa da revista Chemistry: A European Journal (Figura 8) e deu origem a várias entrevistas para a rádio e televisão devido à intensa discussão que tem existido sobre as alterações climáticas e o papel do $\mathrm{CO}_{2}$ [14]. A equipa desfrutou deste

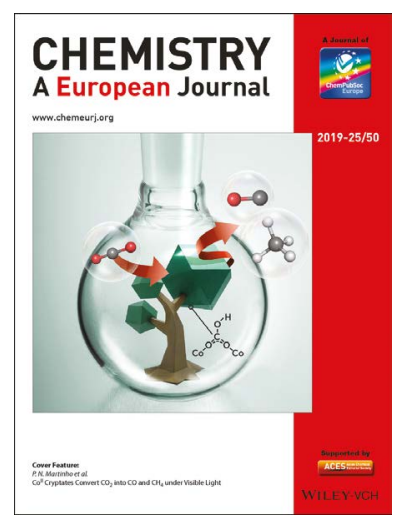

Figura 8 - Capa da revista Chemistry: A European Journal da editora Wiley-VCH [15] 
contacto mais direto com a comunicação de ciência à sociedade, o que fez com que se queira empenhar ainda mais no desenvolvimento da ciência em Portugal.

Os resultados obtidos neste trabalho demonstram a importância da preparação de compostos de coordenação, neste caso criptatos, que são capazes de mimetizar processos existentes na natureza. Estes criptatos deram origem a um sistema sustentável que é ativado por luz visível para a conversão de $\mathrm{CO}_{2}$ em CO e $\mathrm{CH}_{4}$, que são produtos que podem ser usados como alternativa aos combustíveis fósseis. Como se de um ciclo energético viável se tratasse, usa-se um poluente para gerar moléculas que satisfazem as necessidades da sociedade que, após o seu consumo, geram novamente $\mathrm{CO}_{2}$, que pode ser reconvertido. Este trabalho deu origem a uma nova linha de investigação onde se pretende otimizar a estrutura destes criptatos para a produção apenas de $\mathrm{CH}_{4}$, numa reação ativada pela luz visível.

\section{Agradecimentos}

Quero agradecer aos meus orientadores da tese de doutoramento, Doutor Paulo Martinho, Professora Maria José Calhorda e Professora Ana Margarida Martins, especialmente ao primeiro, pelo apoio demonstrado ao longo desta tese. Sem dúvida que o seu apoio contribuiu para os bons indicadores que resultaram desde trabalho do qual muito me orgulho. Quero agradecer também ao programa doutoral "Catálise e Sustentabilidade" (CATSUS) pela bolsa de doutoramento atribuída (PD/BD/52368/2013).

\section{Referências}

[1] M. A. Scibioh, B. Viswanathan, "Carbon Dioxide to Chemicals and Fuels", Elsevier, 2018.

[2] NASA, "Graphic: The relentless rise of carbon dioxide": climate.nasa.gov/ vital-signs/carbon-dioxide (acedido em 03/02/2020).

[3] M. Aresta, Coord. Chem. Rev. 2016, 113, 6621-6658. D0I: 10.1021/cr300463y.

[4] C. Finn, S. Schnittger, L. J. Yellowlees, J. B. Love, Chem. Comm. 2012, 48, 1392-1399. DOI: 10.1039/C1CC15393E.

[5] J. Schneider, H. Jia, J. T. Muckerman, E. Fujita, Chem. Soc. Rev. 2012, 41, 2036-2051. DOI: 10.1039/C1CS15278E.

[6] H. Takeda, C. Cometto, 0. Ishitani, M. Robert, ACS Catal. 2017, 7, 70-88. DOI: 10.1021/acscatal.6b02181.

[7] J. M. Lehn, Pure Appl. Chem. 1978, 50, 871-892. DOI: 10.1351/ pac197850090871.

[8] Y. Dussart, C. Harding, P. Dalgaard, C. McKenzie, R. Kadirvelraj, V. McKee, J. Nelson, J. Chem. Soc. Dalton Trans. 2002, 1704-1713. D0I: 10.1039/B110449G.

[9] C. T. Supuran, Nat. Rev. Drug Discov. 2008, 7, 168-181. DOI: 10.1038/nrd2467.
[10] F. Möller, L. Castañeda-Losada, J. R. C. Junqueira, R. G. Miller, M. L. Reback, B. Mallick, M. van Gastel, U.-P. Apfel, Dalton Trans. 2017, 46, 5680-5688. DOI: 10.1039/C6DT04527H.

[11] F. Möller, K. Merz, C. Herrmann, U.-P. Apfel, Dalton Trans. 2016, 45, 904-907. DOI: 10.1039/C5DT04267D.

[12] Sara Realista, Sequestration and reduction of $\mathrm{CO}_{2}$ using multifunctional metalorganic materials. Tese de Doutoramento em Química. Universidade de Lisboa, 2018. bibliotecas.utl.pt/cgi-bin/koha/opac-detail. pl?biblionumber $=524827$.

[13] T. Ouyang, H. H. Huang, J. W. Wang, D. C. Zhong, T. B. Lu, Angew. Chem. Int. Ed. 2017, 56, 738-743. DOI: 10.1002/anie.201610607.

[14] S. Realista, J. C. Almeida, S. A. Milheiro, N. A. G. Bandeira, L. G. Alves, F. Madeira, M. J. Calhorda, P. N. Martinho, Chem. Eur. J. 2019, 25, 11670-11679. DOI: 10.1002/chem.201901806.

[15] S. Realista, J. C. Almeida, S. A. Milheiro, N. A. G. Bandeira, L. G. Alves, F. Madeira, M. J. Calhorda, P. N. Martinho, Chem. Eur. J. 2019, 25, 11583-11583. DOI: 10.1002/chem.201903441.

\section{Sara Realista}

Instituto de Tecnologia Química e Biológica António Xavier, ITQB NOVA, Universidade Nova de Lisboa, Oeiras, Portugal. Investigadora no projeto Chemical Synthesis Using Earth-Abundant Metal Catalysts no grupo de Catálise Organometálica liderado pela Dra. Beatriz Royo no Instituto de Tecnologia Química e Biológica. Doutorou-se em 2018 e tem 13 artigos em revistas científicas internacionais e dois prémios nacionais. O seu principal interesse é a procura de novos compostos e metodologias para um mundo sustentável.

sara.realista@itqb.unl.pt ORCID.org/0000-0003-1066-1200 